POS $\quad$ PROCEEDINGS

\title{
Geometrical invariants, conserved currents and new symmetries in a covariant phase-space dynamics
}

\section{Luis A. Cabral}

UFT, Araguaina-TO, Brazil

E-mail: cabraleuft.edu.br

\begin{abstract}
We consider a model that describes a charged particle in curved spacetime with external gauge fields by means of a recently proposed covariant phase-space formalism. In this formalism, which is more suited to find invariants, the phase-space brackets are changed to an extended algebra containing the field strength of the gauge fields. However the contribution from the electromagnetic interaction will alter the set of the Killing tensor equations in order to obtain some conserved quantities. With the Killing tensor still there is a geometric duality that implies in a completely non-trivial metric associated to the spacetime. Using this metric some geometrical invariants and currents are considered.
\end{abstract}

5th International School on Field Theory and Gravitation, April 20 - 242009

Cuiabá city, Brazil 


\section{Introduction}

The symmetries in curved spacetime was intensively investigated a long time ago and is still the subject of many applications related to integrability of geodesics motion, separability of Hamilton-Jacobi and scalar field equations, higher dimensional black-holes and much more (see a recent review [1] and references therein). An important fact concerning geodesics is with respect with their associated isometries. These are generated by Killing vector fields and with these fields conserved quantities are constructed along the geodesic motion. The extension of isometries using higher rank tensors can be considered in the phase space representation of the geodesic motion by means of a particle in curved space. In this case, conserved quantities, in the Hamiltonian formalism, can be described with multiples of a particle momenta contracted with symmetric Killing tensors of higher rank [2]. The symmetries in phase space associated with these conserved quantities, are also known as hidden symmetries in spacetime since are not directly present in the metric transformations. Besides symmetry property, there is the duality one. A system that is dual to another can retain some properties in a simplified (or non-trivial) formulation but described with different variables or parameters. However, in the phase space analysis, it was proposed a new kind of duality [2] known as geometric duality. Using this one we consider how the phase space symmetries for a charged particle in curved spacetime can determine a new kind of dual metric. From this metric conserved currents and geometrical invariants will be considered beyond other subjects that will be discussed in the following sections.

\section{Symmetries and Killing Tensors}

When we consider an isometry, it is represented by the well known equation for a Killing vector $\xi^{\mu}$

$$
\nabla_{(\mu} \xi_{v)}=0
$$

We can consider a generalization of (2.1) for objects of higher rank, i.e., for tensorial fields defined in the spacetime. In this case, we have

$$
\begin{aligned}
& \nabla_{(\mu} \xi_{\left.v_{1} v_{2} \cdots v_{n}\right)}=0, \\
& \nabla_{(\mu} f_{\left.v_{1}\right) v_{2} \cdots v_{n}}=0,
\end{aligned}
$$

where in (2.2) we have a definition of a Killing tensor $\xi_{\mu_{1} \mu_{2} \cdots \mu_{n}}$ of order $n>1$, with $\xi_{\left(v_{1} v_{2} \cdots v_{n}\right)}=$ $\xi_{v_{1} \cdots v_{n}}$, which represents a $n$ order totally symmetric tensor. Analogously, for an antisymmetric tensor, we define in (2.3) a Killing-Yano Tensor $f_{\mu_{1} \mu_{2} \cdots \mu_{n}}$ with $f_{\mu_{1} \mu_{2} \cdots \mu_{n}}=f_{\left[v_{1} v_{2} \cdots v_{n}\right]}$.

An important property of the Killing Tensor object (2.3) appears when we use a standard Hamiltonian for a neutral particle in curved space $H=\frac{1}{2} p_{\mu} p_{\nu} g^{\mu \nu}(x)$, which implies in a constant of motion given by $\xi_{(2)}=\frac{1}{2} \xi^{\mu v} p_{\mu} p_{v}$, since its time evolution is given by

$$
\begin{aligned}
\left\{\xi_{(2)}, H\right\} & =\left\{p_{\mu} p_{\nu} \xi^{\mu v}, p_{\lambda} p_{\rho} g^{\lambda \rho}\right\} \\
& =\frac{2}{3} p^{\alpha} p^{\beta} p^{\lambda} \nabla_{(\lambda} \xi_{\alpha \beta)}=0,
\end{aligned}
$$


where $\xi_{\alpha \beta}$ is a symmetric second rank Killing tensor and it was used the standard Poisson brackets.

We can interpret from the equation (2.4) that we can have a Hamiltonian in a spacetime with metric $\xi^{\mu v}$ and a Killing tensor $g^{\alpha \beta}$, or even, in the original form, a spacetime with metric $g^{\mu v}$ and Killing tensor $\xi^{\lambda \beta}$. This dual aspect is known as geometric duality, as proposed in [2]. In this aspect is defined a contravariant dual metric $\tilde{g}^{\mu v}$ given by

$$
\tilde{g}^{\mu \nu}=\xi^{\mu \nu}=g^{\mu \alpha} \xi_{\alpha \rho} g^{\rho v} .
$$

If this contravariant metric is non-degenerate, we can consider its inverse by $\tilde{g}_{\mu v}$, since

$$
\tilde{g}^{\mu \rho} \tilde{g}_{\rho v}=\delta_{v}^{\mu}
$$

\section{The Covariant Dynamics and Extended Geometric Duality}

If we consider the Hamiltonian of a charged particle moving in a space with metric $g_{\mu \nu}$ interacting with an external gauge field $A_{\mu}$,

$$
H=\frac{1}{2 m} g^{\mu v} \Pi_{\mu} \Pi_{v}
$$

where

$$
\Pi_{\mu}=p_{\mu}-q A_{\mu}
$$

is a gauge invariant momenta, the dynamics is now manifest in the modified brackets [3]

$$
\{f, g\}_{C}=\frac{\partial f}{\partial x^{\mu}} \frac{\partial g}{\partial \Pi_{\mu}}-\frac{\partial f}{\partial \Pi_{\mu}} \frac{\partial g}{\partial x^{\mu}}+q F_{\mu v} \frac{\partial f}{\partial \Pi_{\mu}} \frac{\partial g}{\partial \Pi_{v}}
$$

for phase-space functions $f\left(x^{\mu}, \Pi_{v}\right) ; g\left(x^{\mu}, \Pi_{v}\right)$ and supplemented with the field-strenght $F_{\mu v}$. This formulation is useful to find invariants in phase-space, as obtained in [3], since the standard Hamiltonian (using exclusively canonical momenta) is not appropriated for this goal. An application of this formulation could be useful in the model proposed in [4] to find possible relations between invariants and experimental measurements.

Using (3.3), we consider

$$
\begin{aligned}
\left\{\xi_{(2 C)}, H\right\}_{C} & =\left\{\Pi_{\mu} \Pi_{v} \xi^{\mu v}, \Pi_{\lambda} \Pi_{\rho} g^{\lambda \rho}\right\}_{C} \\
& =\frac{2}{3} \Pi^{\alpha} \Pi^{\beta} \Pi^{\lambda} \nabla_{(\lambda} \xi_{\alpha \beta)}+q \Pi^{\alpha} \Pi^{\beta} \xi_{\rho(\beta} F_{\alpha)}{ }^{\rho}=0,
\end{aligned}
$$

and we can see that there are additional conditions to consider a geometric duality in the covariant phase-space dynamics.

The Generalized Killing Tensor equation, in the sense of (2.5), to solve will be (3.5), and now this equation with an additional condition on the Killing Tensor, will imply in a new kind of dual metric and probably in new invariants in the covariant phase space.

Firstly, as a particular case, we consider the Kimura metric (for a recent use of this metric see [6]) and consider it in the equations (2.2) for $n=2$ in order to find the Killing tensor compatible with the set of equations in (2.5). 
From the Kimura metric represented by

$$
d s^{2}=\frac{r^{2}}{b} d t^{2}-\frac{1}{r^{2} b^{2}} d r^{2}-r^{2}\left(d \theta^{2}+\sin ^{2} \theta d \phi^{2}\right),
$$

we obtain the second rank Killing tensor $\xi_{\mu v}$, whose nonzero components are given by:

$$
\begin{aligned}
\xi_{11}(r, \theta)= & \left(-c_{2} \frac{b}{r^{2}}+c_{3}\right) r^{4} \\
\xi_{22}(r, \theta)= & c_{2} / r^{2} \\
\xi_{33}(r, \theta)= & \left(c_{2} \frac{b^{2}}{r^{2}}+c_{4}\right) r^{4} \\
\xi_{41}(r, \theta)= & -\frac{1}{2} r^{4} c_{1}(-1+\cos 2 \theta), \\
\xi_{44}(r, \theta)= & -\frac{1}{2} c_{2} r^{2} b^{2}(-1+\cos 2 \theta) \\
& +r^{4}\left(-\frac{1}{16} c_{5}(10-15 \cos 2 \theta+6 \cos 4 \theta-\cos 6 \theta) /(-1+\cos 2 \theta)\right. \\
& -\frac{1}{4} c_{4}(3+\cos 4 \theta-4 \cos 2 \theta) /(-1+\cos 2 \theta),
\end{aligned}
$$

where $c_{i}\left(i=1, \cdots, c_{7}\right)$ are constants.

The Kimura metric is the only one to imply in an irreducible second rank Killing tensor (nondegenerate) obtained by a contraction of third rank Killing Yano tensors [6]. This fact motivate us to consider this metric in our calculations.

At this point is important to observe that if we consider the dynamics in terms of a covariant bracket, the Killing tensor to be obtained must obey an additional condition,

$$
F_{\alpha \beta} g^{\beta \rho} \xi_{\rho \lambda}+F_{\lambda \beta} g^{\beta \rho} \xi_{\rho \alpha}=0,
$$

which belongs to the second term in (3.5).

Considering in general form a gauge field

$$
A_{\mu}=(f(r, \theta), g(r, \theta), h(r, \theta), i(r, \theta))
$$

a nontrivial solution of (3.5), among a lot of others possible solutions according to integral constants, is given by

$$
\begin{aligned}
\xi_{11}(r, \theta) & =\left(-c_{2} \frac{b}{r^{2}}+c_{3}\right) r^{4}, \\
\xi_{22}(r, \theta) & =c_{2} / r^{2}, \\
\xi_{33}(r, \theta) & =\left(c_{2} \frac{b^{2}}{r^{2}}+c_{4}\right) r^{4}, \\
\xi_{41}(r, \theta) & =r^{4} c_{1} \sin ^{2} \theta, \\
i(r, \theta) & =\frac{\left(b c_{3}+c_{4}\right) F_{1}(\theta)}{c_{1}}+c_{5}, \\
\xi_{44}(r, \theta) & =\frac{r^{2}}{b c_{3}+c_{4}}\left(r^{2} b^{2} c_{1}{ }^{2}\left(\frac{\cos ^{2} 2 \theta}{4}-\frac{\cos 2 \theta}{2}+\frac{1}{4}\right)\right.
\end{aligned}
$$




$$
\begin{aligned}
& \left.+\left(b c_{3}+c_{4}\right)\left(b^{2} c_{2}+c_{4} r^{2}\right) \sin ^{2} \theta\right), \\
h(r, \theta)= & \int \frac{\partial g(r, \theta)}{\partial \theta} d r+F_{2}(\theta), \\
f(r, \theta)= & F_{1}(\theta)
\end{aligned}
$$

where $g(r, \theta), F_{1}(\theta)$ and $F_{2}(\theta)$ are arbitrary functions, and $c_{i}(i=1, \cdots, 7)$ are constants.

With the additional restriction (3.12), we can note that there will be more parameters to choose in order to furnish a final form of a dual metric in the covariant phase-space formulation. In this way we can obtain many classes of dual metrics according to the electromagnetic field interaction.

\section{Conserved currents and geometrical invariants from dual space}

Since from a second rank symmetric Killing-Tensor we can obtain many classes of dual metrics, we discuss what kind of antisymmetric object could be obtained from an antisymmetric Killing-Yano tensor of the same order.

For this case we note the existence of another conserved quantity, but now related with the Killing-Yano Tensor as found in [5]. Let us consider a particular case of rank 2, antisymmetric Killing-Yano Tensor, however in the dual metric space.

In this case, since the spacetime admits a Killing-Yano Tensor, we can construct a dual current given by

which satisfies

$$
\tilde{j}^{\alpha \beta}=-\frac{1}{4}\left(\tilde{f}^{\gamma \lambda} \tilde{R}_{\gamma \lambda}^{\alpha \beta}-2 \tilde{f}^{\alpha \gamma} \tilde{R}_{\gamma}^{\beta}+2 \tilde{f}^{\beta \gamma} \tilde{R}_{\gamma}^{\alpha}+\tilde{f}^{\alpha \beta} \tilde{R}\right)
$$

$$
\nabla_{\alpha} \tilde{j}^{\alpha \beta}=0
$$

by means of the following identities:

$$
\nabla_{[\alpha} \tilde{R}_{\beta \gamma] \lambda \varepsilon}=0, \quad \nabla_{\alpha} \tilde{R}_{\beta \gamma \lambda}^{\alpha}+2 \nabla_{[\beta} \tilde{R}_{\gamma] \lambda}=0, \quad \nabla_{\alpha} \tilde{R}_{\beta}^{\alpha}-\frac{1}{2} \nabla_{\beta} \tilde{R}=0 .
$$

We note in (4.1) the use of dual metric, and the objects that follow from it. This expression is a huge one, since involve a lot of terms from dual part. The physical interpretation of this expression in dual space still needs exploration.

Another point that still needs attention is the singularity behaviour of the dual metric space. A geometrical invariant that explore this behaviour is the Kretschmann invariant,

$$
\tilde{K}=\tilde{R}^{\mu \nu \lambda \rho} \tilde{R}_{\mu \nu \lambda \rho},
$$

that in this case is obtained by the full contraction of the dual Riemann tensor, in the sense that it was obtained from Riemann tensor defined in terms of the dual metric. Another geometrical invariant could be explored to perform the horizon behaviour in the dual space and will be considered elsewhere. It is important to note, that for the nontrivial dual Kimura metric, the determinant $\tilde{g}$ of the dual metric is a large expression, and implies in a degenerate metric if $c_{2}=0$ as we can see in the following:

$$
\begin{aligned}
\tilde{g}= & -\sin ^{2} \theta c_{2} r^{4}\left(c_{2} b^{2}+c_{4} r^{2}\right)\left(c_{2}^{2} b^{3}-c_{3} c_{2} b^{2} r^{2}-c_{2} b c_{5} \cos ^{2} \theta r^{2}+c_{2} b c_{4} r^{2}\right. \\
& \left.+c_{2} b c_{5} r^{2}+c_{3} c_{5} \cos ^{2} \theta r^{4}-c_{1}^{2} \cos ^{2} \theta r^{4}-c_{3} c_{5} r^{4}-c_{3} c_{4} r^{4}+c_{1}^{2} r^{4}\right) .
\end{aligned}
$$


From this expression we can infer a huge extension of the Kretschmann geometrical invariant (4.4). The details of this analysis, as well applied to other categories of metrics, will be reported elsewhere.

Finally the possible interchange between $\xi_{\mu \nu}$ and $f_{\mu \nu}$ in the geometric duality will be the subject of future investigations, beyond the influence in the conserved currents and geometrical invariants in dual spaces.

\section{Acknowledgments}

The author would like to thanks useful discussions with João E. Frederico that helps to improve the work. Thanks also to the Organizing Committee of the 5th ISFTG for a successful event.

\section{References}

[1] V. P. Frolov and D. Kubiznak, Class. Quant. Grav. 25 (2008) 154005.

[2] R. H. Rietdijk and J. W. van Holten, Nucl. Phys. B472, 427 (1996).

[3] J. W. van Holten, Phys. Rev. D75 (2007) 025027.

[4] L. A. Cabral, G. Gusev and N. M. Sotomayor, Classical magnetoresistance of a two-dimensional electron gas constrained to non-planar topographies in antidot lattice under tilted magnetic field AIP conference proceedings, USA, V. 893 (2007) 563-564.

[5] D. Kastor and J. Traschen, JHEP 0408 (2004) 045.

[6] F. C. Popa and O. Tintareanu-Mircea, Mod. Phys. Lett. A22 (2007) 1309-1318. 